\title{
Quenched and negative Hall effect in periodic media: application to antidot superlattices
}

\author{
R. Fleischmann ${ }^{1}$, T. Geisel ${ }^{1}$, and R. Ketzmerick ${ }^{1,2}$ \\ ( $\left.{ }^{1}\right)$ Institut für Theoretische Physik und Sonderforschungsbereich Nichtlineare Dynamik, \\ Universität Frankfurt, D-60054 Frankfurt/Main, Federal Republic of Germany \\ $\left({ }^{2}\right)$ Physics Department, University of California, Santa Barbara, CA 93106, USA
}

\begin{abstract}
We find the counterintuitive result that electrons move in opposite direction to the free electron $\mathbf{E} \times \mathbf{B}$-drift when subject to a two-dimensional periodic potential. We show that this phenomenon arises from chaotic channeling trajectories and by a subtle mechanism leads to a negative value of the Hall resistivity for small magnetic fields. The effect is present also in experimentally recorded Hall curves in antidot arrays on semiconductor heterojunctions but so far has remained unexplained.
\end{abstract}

PACS numbers: 73.40.L, 05.45, 73.20.M

Europhysics Letters, in press.

Typeset using REVTEX 
We have long become used to the fact, that the Hall resistance of extended systems grows linearly with the magnetic field $B$ apart from quantum phenomena like the quantum Hall effect. It thus came as a surprise when for small $B$ in antidot superlattices the Hall resistance remained quenched and even took on negative values [1, 2] although the current is carried by electrons and not by holes. In spatially constricted systems like ballistic microjunctions of crossed wires similar phenomena had previously been reported [3 5] and were attributed to ballistic rebound trajectories, where after colliding with a confining wall the electron is ejected into the opposite lead [1, 6-8]. Since this explanation rests upon the finiteness and smallness of the junction, it cannot account for large and extended systems. Thus the negative Hall resistance in antidot superlattices (with as many as $10^{5}$ lattice cells) has remained mysterious and one may have wondered whether it is not an artifact or an edge effect.

In the present paper we describe a general mechanism whereby the presence of a periodic superlattice potential causes a majority of electrons to move in a direction opposite to the free electron $\mathbf{E} \times \mathbf{B}$-drift. It gives rise to quenching and negative Hall resistivity as a bulk property. In the regime of small magnetic fields there is a long-time tail associated with chaotic channeling trajectories, which tend to follow one of the channels of the 2D potential as they are trapped close to a tube of regular motion in phase space. The escape from these channels after crossing 3 to 1000 or more lattice cells is predominantly in opposite direction to the Lorentz force. The latter phenomenon bears a partial resemblance to the rebound trajectories responsible for quenching in ballistic microjunctions, where in a sense one deals with a single unit cell of an antidot lattice. However, it is the combination of the longtime tail and the asymmetric escape that gives rise to the negative Hall effect in periodic media, as one can see from two arguments. Rebound trajectories can have a larger curvature $($ radius $\approx a)$ and would occur up to much higher magnetic fields $\left(B / B_{0} \approx 0.5\right.$ in Fig. (3) $)$ where quenching would be predicted but has disappeared in our extended system. This is the regime of magnetoresistance $\left(\varrho_{x x}\right)$ oscillations due to cyclotron-type orbits [1, 9] where the Hall resistivity $\left(\varrho_{x y}\right)$ exhibits other features (Fig. 3), which are also in good quantitative 
agreement with experiment. Secondly, we find that the negative Hall resistivity is absent for infinitely steep antidots (Sinai billiard) where only a few channeling trajectories exist, whereas the rebound mechanism would be compatible with steep walls. Furthermore the quenching mechanism is not an edge but a bulk effect as it shows up even in the Hall resistivity and not only in the resistance.

In microjunctions and lateral surface superlattices the dynamics of electron wave packets approaches the classical limit as the Fermi wavelength is smaller than the typical length scale of the lateral structure. We model the antidot superlattice with lattice constant $a$ by the continuous potential [9] (Fig. 11 right inset)

$$
U(x, y)=U_{0}[\cos (\pi x / a) \cos (\pi y / a)]^{\beta},
$$

with $\beta=4$. The prefactor $U_{0}$ of the potential is chosen such that the ratio of the dot diameter at the Fermi energy $\varepsilon_{F}$ to the distance of adjacent dots is one-third, as in the experiments [1]. The classical approximation for the dynamics of an electron wave packet in the potential $U(x, y)$ and a perpendicular magnetic field $\mathbf{B} \| \mathbf{e}_{z}$ is then given by 4 coupled differential equations, which in scaled variables $\left(x \rightarrow x / a, y \rightarrow y / a, U \rightarrow U / \varepsilon_{F}, t \rightarrow t / t_{0}\right.$, with $\left.t_{0}=\left(\varepsilon_{F} / m a^{2}\right)^{1 / 2}\right)$ read

$$
\begin{aligned}
& \dot{x}=v_{x} \quad \dot{v}_{x}=2 \sqrt{2}\left(B / B_{0}\right) v_{y}-\partial U / \partial x \\
& \dot{y}=v_{y} \quad \dot{v}_{y}=-2 \sqrt{2}\left(B / B_{0}\right) v_{x}-\partial U / \partial y
\end{aligned}
$$

A chaotic trajectory for small magnetic field $B / B_{0}=0.1$ is shown in Fig. 1, where $B_{0}=$ $2\left(2 m \varepsilon_{F}\right)^{1 / 2} /(e a)$ corresponds with a free cyclotron radius of $a / 2$ of an electron with effective mass $m$. The trajectory follows one of the channels in the potential interrupted by local chaotic motion. Thus the free cyclotron motion in a magnetic field is destroyed by the periodic potential and the electron is forced into long episodes of linear motion. This is visualized in more detail in the left inset of Fig. 11.

We investigate the motion in phase space $\left(x, y, v_{x}, v_{y}\right)$ for $B \neq 0$ and $E=0$, by means of Poincaré surfaces of section $\left(x, v_{x}\right)$ at $y(\bmod 1)=0$ for various initial conditions. In Fig. 2 for 
$v_{y}>0$ (left) and $v_{y}<0$ (right), this section displays a sea of chaotic motion and an island of regular motion which represents a tube of trajectories channeling forever in the $y$-direction. It contains periodic and quasiperiodic trajectories lying on cylindrical KAM-surfaces [10] around a central trajectory in phase space. They arise from nonlinear resonances between the degrees of freedom and are represented by closed loops in the Poincaré surface of section. In the neighbourhood of such a tube of regular channeling trajectories, a chaotic trajectory can be trapped in an infinite hierarchy of permeable barriers, so-called cantori (not visible in Fig. 2). This appears to be the origin of the long channeling episodes of the chaotic trajectory in Fig. 1. They are associated with a long-time tail as in similar systems which we previously studied [11,12]. Here they will turn out to be essential for the negative Hall effect. We note that the tubes of regular motion in both directions $\left(v_{y}>0\right.$ and $\left.v_{y}<0\right)$ as well as surrounding chaotic channeling trajectories still exist in a weak electric field $\mathbf{E} \| \mathbf{e}_{x}$. This means that many trajectories move in opposite direction to the free $\mathbf{E} \times \mathbf{B}$-drift and qualitatively explains the quenching of the Hall effect. For a more detailed account we now apply a linear response theory including regular as well as chaotic trajectories.

We therefore divide phase space into regions of chaotic and regular motion corresponding to Fig. 2 and write the conductivity $\boldsymbol{\sigma}$ as

$$
\boldsymbol{\sigma}=p_{r} \boldsymbol{\sigma}^{r}+p_{c} \boldsymbol{\sigma}^{c}
$$

where $p_{r}$ and $p_{c}$ are the portions of phase space volume occupied by regular and chaotic trajectories, respectively. Drude-type considerations for the straight regular trajectories in the channels lead to longitudinal conductivities $\sigma_{x x}^{r}=\sigma_{y y}^{r}=p_{r} n \tau e^{2} / 2 m$, where $n$ is the electron density and $\tau$ is the mean elastic scattering time, and to vanishing transversal conductivities $\sigma_{x y}^{r}=\sigma_{y x}^{r}=0$. Combining this with the conductivity of chaotic trajectories as derived in Ref. [9] using linear response theory yields

$$
\sigma_{i j}=p_{r} \sigma_{i j}^{r}+\left(1-p_{r}\right) \sigma_{0} \int_{0}^{\infty} e^{-\frac{t}{\tau}} C_{i j}(t) d t
$$

where $C_{i j}(t)=\left\langle v_{i}(t) v_{j}(0)\right\rangle$ is the correlation function of the unperturbed chaotic trajectories (i.e. neither perturbed by an electric field nor by impurity scattering ) and the factor 
$\exp (-t / \tau)$ describes impurity scattering [13]. The numerical analysis involves the determination of $p_{r}$ as the volume of the outermost invariant KAM-surface enclosing the regions of regular motion divided by the total phase space volume. As these regular regions are small, however, the main contribution to the conductivities calculated according to Eq. (丑) stems from chaotic trajectories, whose correlation functions $C_{i j}(t)$ were determined from numerical simulations. The resulting Hall resistivity $\varrho_{x y}$ (Fig. 3) shows a quenching of the Hall effect and even a negative Hall resistivity as in the experiment [1]. The negative Hall resistance in antidot superlattices thus is a bulk effect due to chaotic electron trajectories.

We now analyze the dynamical features leading to a negative Hall effect at small magnetic fields. The sign of the Hall resistivity $\varrho_{x y}=\sigma_{y x} /\left(\sigma_{x x}^{2}+\sigma_{y x}^{2}\right)\left(\right.$ with $\left.\sigma_{x y}=-\sigma_{y x}\right)$ is given by the sign of $\sigma_{y x}$, which according to Eq. (4) is determined essentially by the correlation function $C_{y x}(t)$ of the chaotic trajectories. Figure $\$$ shows the time dependence of $C_{y x}(t)$ for $B / B_{0}=0.05$ where a negative Hall resistivity was found. Its initial increase still follows the free electron case but for later times there is a strong negative peak and a negative algebraic long time tail. This tail is responsible for the negative Hall effect as it yields a dominance of negative contributions in the integral over time in Eq. (四). Note that the mean elastic scattering time is $\tau \approx 25$ in the experiment [四]. For larger magnetic fields $\left(B / B_{0} \geq 0.12\right.$, see dotted line in Fig. (4) the tail disappeares and $C_{y x}(t)$ exhibits an oscillatory decay where positive contributions to $\sigma_{y x}$ progressively outweigh the negative ones. The oscillatory decay for $B / B_{0} \geq 0.12$ is caused by chaotic trajectories that roughly follow cyclotron-type orbits around 1 or 4 antidots for a limited time and were shown to be responsible for pronounced peaks in the magnetoresistance $\varrho_{x x}(B)$ (Ref. [9] and dotted line in the inset of Fig. 3). Many rebound trajectories [4,6,7] would have a similar curvature and would be expected in this magnetic field range $\left(B / B_{0} \approx 0.5\right)$, yet they do not lead to a quenched or negative Hall effect. Instead one finds a prominent Hall plateau, which corresponds well with the experimental results [1, 14, 15]. In the experiment of Ref. [1], e.g. $B_{0} \approx 0.55 \mathrm{~T}$.

The long-time tail stems from chaotic channeling trajectories, which exhibit long episodes of straight motion (Fig. 1) and dominate the dynamics below $B / B_{0}=0.12$. The distribution 
$\psi(\ell)$ of channeling lengths decays algebraically $\psi(\ell) \propto \ell^{-\nu}$ where $\nu$ fluctuates around $\nu=3$. Long-time tails seem to be typical for chaotic Hamiltonian systems and are believed to be related to cantorus hierarchies (see e.g. Ref. [16,11) as described in the context of Fig. 2, but their origins are still not understood definitively. In the present case, after moving in a channel e.g. along the $x$-axis for a while (at least for 3 unit cells) the trajectories tend to leave the channel in the direction opposite to the Lorentz force $-e \mathbf{v} \times \mathbf{B}$ (which points to their left). For example at $B / B_{0}=0.05$ a majority of about $65 \%$ of the trajectories show this anomaly (see Fig. 1 inset) and thereby yield the negative sign of the long-time tail. Intuitively the experimental situation can be imagined by an applied current transported mainly by channeled (chaotic) electrons moving in positive $x$-direction. Since they tend to leave the channel in negative $y$-direction they build up a negative Hall voltage.

The role of the chaotic channeling trajectories for the long-time tail and the negative Hall effect can be described in more detail. For times $t \gg a / v_{F}$ and small magnetic fields the decaying correlation function $C_{y x}(t)$ is determined by long channeling episodes and can be expressed analytically in terms of the length distribution $\psi(\ell)$ by [17]

$$
C_{y x}(t) \sim \frac{2 \alpha v_{F}^{2}}{\langle\ell\rangle} \int_{v_{F} t}^{\infty} \psi(\ell)\left[p_{+}(\ell)-p_{-}(\ell)\right] d \ell
$$

where $v_{F}$ is the Fermi velocity, $\alpha v_{F}^{2}$ is the mean absolute value of the contribution to the correlation function of individual channeling episodes, and $\langle\ell\rangle$ is the mean length of these episodes. $p_{+}(\ell)$ and $p_{-}(\ell)$ are the conditional probabilities that a trajectory trapped in a channeling episode of length $\ell$ leaves the channel in positive or negative direction with respect to the Lorentz force. The asymptotic decay of $C_{y x}(t)$ obtained form Eq. (5) is indicated by the dashed line in Fig. 4 . Here $\alpha$ and $p_{+}(\ell)-p_{-}(\ell)$ were obtained approximately from geometrical considerations, while for the length distribution $\psi(\ell)$ we used the numerical data. The long-time tail of $C_{y x}(t)$ is relevant for the negative Hall resistivity up to times $t \approx \tau$, i.e. $\ell \approx 30$. The dashed line in Fig. 1 demonstrates that it is caused by the distribution $\psi(\ell)$ of chaotic channeling lengths.

We emphasize that the results presented here do not depend sensitively on a particular 
choice for the periodic potential. While for an infinitely steep antidot potential the effects are absent, realistic potentials with a small degree of softness produce a negative tail in $C_{y x}(t)$ (we have checked this e.g. for $\beta=20$ in Eq. (1). For the sample used in Ref. [1] $\beta=4$ seems realistic [9]). The strength of the tail determines whether Eq. (4) yields a negative Hall resistivity or only quenching. Furthermore irregularities of the potential lead to a reduction of the tail. (For a distorted potential with $\pm 5 \%$ disorder in the antidot positions we still found a negative Hall resistivity.) This might explain why in some experimental situations quenching and in others a negative Hall effect was reported [1,2].

We acknowledge motivating discussions with R. R. Gerhardts, K. v. Klitzing, and D. Weiss. This work was supported by the Deutsche Forschungsgemeinschaft. RK thanks Walter Kohn for the hospitality in Santa Barbara and for partial support by the NSF under DMR90-01502. 


\section{REFERENCES}

[1] D. Weiss, M. L. Roukes, A. Menschig, P. Grambow, K. v. Klitzing, and G. Weimann, Phys. Rev. Lett. 66, 2790 (1991).

[2] A quenched but positive Hall effect was observed in R. Schuster, K. Ensslin, J. P. Kotthaus, M. Holland, and S. P. Beaumont, Superlattices and Microstructures 12, 93 (1992), Ref. 17

[3] M. L. Roukes, A. Scherer, S. J. Allen, H. G. Craighead, R. M. Ruthen, E. D. Beebe, and J. P. Harbison, Phys. Rev. Lett. 59, 3011 (1987); K. L. Shepard, M. L. Roukes, and B. P. Van der Gaag, Phys. Rev. Lett. 68, 2660 (1992).

[4] C. J. B. Ford, S. Washburn, M. Büttiker, C. M. Knoedler, and J. M. Hong, Phys. Rev. Lett. 62, 2724 (1989).

[5] M. Wendel, C. Lettau, W. Hansen, J. P. Kotthaus, and G. Weimann, to be published.

[6] C. W. J. Beenakker and H. van Houten, Phys. Rev. Lett. 63, 1859 (1989).

[7] H. U. Baranger, D. P. DiVincenzo, P. A. Jalabert, and A. D. Stone, Phys. Rev. B 44, 10637 (1991).

[8] T. Geisel, R. Ketzmerick, and O. Schedletzky, Phys. Rev. Lett. 69, 1680 (1992).

[9] R. Fleischmann, T. Geisel, and R. Ketzmerick, Phys. Rev. Lett. 68, 1367, (1992).

[10] See e.g. V. I. Arnol'd Mathematical Methods of Classical Mechanics (Springer, New York, 1989).

[11] T. Geisel, A. Zacherl, and G. Radons, Phys. Rev. Lett. 59, 2503 (1987); T. Geisel, A. Zacherl, and G. Radons, Z. Phys. B 71, 117 (1988); A. Zacherl, T. Geisel, J. Nierwetberg, and G. Radons, Phys. Lett. 114, 317 (1986).

[12] T. Geisel, J. Wagenhuber, P. Niebauer, and G. Obermair, Phys. Rev. Lett. 64, 1581 
(1990); J. Wagenhuber, T. Geisel, P. Niebauer, and G. Obermair, Phys. Rev. B 45, 4372, (1992).

[13] The exponential factor describes elastic impurity scattering only in first order. This is sufficient however as the effect of impurity scattering on the correlation decay is small compared to the effect of chaotic scattering.

[14] D. Weiss private communication.

[15] K. Ensslin, K. T. Häussler, C. Lettau, A. Lorke, J. P. Kotthaus, A. Schmeller, R. Schuster, P. M. Petroff, M. Holland, and K. Ploog, in Low-Dimensional Electronic Systems, edited by G. Bauer, F. Kuchar, H. Heinrich (Springer-Verlag, Berlin, 1992), p.45.

[16] J. D. Meiss and E. Ott, Phys. Rev. Lett. 55, 2741 (1985); J. D. Meiss and E. Ott, Physica 20D, 387 (1986);

[17] R. Fleischmann, T. Geisel, and R. Ketzmerick, to be published. 


\section{FIGURES}

FIG. 1. A chaotic trajectory in the antidot potential (right inset) most of the time shows straight paths along a channel even though a perpendicular magnetic field $\left(B / B_{0}=0.1\right)$ is applied. A magnification of such a channeled trajectory in positive and negative $y$-direction (left inset) shows an interplay of left turns due to the magnetic field and of right turns due to deflections from the antidot potential.

FIG. 2. Poincaré surfaces of section at $y(\bmod 1)=0$ for $v_{y}>0$ (left) and $v_{y}<0$ (right) for a weak magnetic field $B / B_{0}=0.1$. The closed curves in the center represent tubes of regular motion intersecting the plane perpendicularly in both directions. The quenching of the Hall effect is related to the survival of these tubes in both directions $\left(v_{y}>0\right.$ and $\left.v_{y}<0\right)$ when a weak electric field $\mathbf{E} \| \mathbf{e}_{x}$ is applied.

FIG. 3. Hall resistivity $\varrho_{x y}$ versus magnetic field $B / B_{0}$ showing a negative Hall effect for $B / B_{0}<0.12$ calculated from Eq. (4). Above $B / B_{0}=0.12$ the inset shows a prominent plateau in $\varrho_{x y}$ for $0.4<B / B_{0}<0.9$ (solid line) caused by chaotic trajectories revolving around 1 or 4 antidots for a while (the dotted line shows $\varrho_{x x}$ after Ref. [9] for comparison). The small plateau in $\varrho_{x y}$ and the shoulder in $\varrho_{x x}$ at $B / B_{0} \approx 0.25$ are due to chaotic trajectories enclosing 2 antidots. (The $\varrho_{x x}$ and $\varrho_{x y}$ scales are not identical.)

FIG. 4. The velocity correlation function $C_{y x}(t)$ for $B / B_{0}=0.05$ (solid line) exhibits a large negative peak and a negative algebraic tail. Both are caused by channeled chaotic trajectories (inset), which tend to leave the channel in a direction opposite to the Lorentz force $-e \mathbf{v} \times \mathbf{B}$ and thus give a negative contribution to the correlation. According to Eq. (4) the area enclosed under $C_{y x}(t)$ up to the elastic scattering time $\tau=25$ [1] is a measure for the Hall conductivity. - Above $B / B_{0}=0.12$ other chaotic trajectories dominate, which revolve around 1 or 4 antidots and thereby produce oscillations in $C_{y x}(t)$ (dotted line for $\left.B / B_{0}=0.15\right)$. The dashed line shows how the length distribution $\psi(\ell)$ according to Eq. (5) gives rise to the tail for $B / B_{0}=0.05$. 